\title{
Técnicas de Geolocalização em Redes LoRaWAN como Abordagem de Tolerância a Falhas para Dispositivos IoT Baseados em GPS
}

\author{
André F. Pastório ${ }^{12}$, Edson T. de Camargo ${ }^{12}$ \\ ${ }^{1}$ Universidade Tecnológica Federal do Paraná (UTFPR) - Toledo - PR - Brasil \\ ${ }^{2}$ Programa de Pós-Graduação em Ciência da Computação (PPGComp) - UNIOESTE - Cascavel - PR - Brasil \\ a.f.pastehotmail.com, edsondutfpr.edu.br
}

\begin{abstract}
Resumo. A ampla adoção do conceito de Internet das Coisas (IoT) e cidades inteligentes passa por aumentar a confiabilidade e a disponibilidade das suas aplicações perante falhas. Redes de longo alcance e baixo consumo de energia são utilizadas em tais aplicações para fornecer conectividade. Nesse contexto, $o$ rastreamento de objetos é uma importante funcionalidade. Normalmente o GPS é utilizados para tal propósito. No entanto, a falha do GPS pode comprometer a aplicação. Este trabalho implementa uma estratégia de geolocalização híbrida em redes LoRaWAN. A implementação é comparada com uma ferramenta comercial, onde um ambiente com três gateways LoRaWAN foi montado para realizar a avaliação. Os resultados demonstram erros de cerca de 151 e 19 metros no pior e melhor caso, respectivamente.
\end{abstract}

\begin{abstract}
The wide adaptation of the concept of Internet of Things (IoT) and smart cities involves increasing the reliability and availability of applications in the face of failures. Long-range, low-power networks are used in such applications to provide connectivity. In this context, object tracking is an important feature. Usually GPS is used for that purpose. However, GPS failure may compromise the application. This work implements a hybrid geolocation strategy in LoRaWAN networks. The implementation is compared to a commercial tool where an environment with three gateways LoRaWAN was set up to perform an evaluation. Results show errors of about 151 and 19 meters in the worst and best case, respectively.
\end{abstract}

\section{Introdução}

A Internet das Coisas (IoT) visa transformar dispositivos comuns do nosso dia a dia em objetos inteligentes ao equipá-los com recursos de identificação, sensoriamento, processamento, comunicação e conexão com a Internet. No contexto de cidades inteligentes, que se apoia no conceito de IoT, estão aplicações nos domínios de transporte público, logística, tráfego inteligente e rastreamento de frotas [Khelifi et al. 2019]. Nessas aplicações, um requisito central é a conectividade onde se destacam as redes de longo alcance e baixa potência, conhecidas como Low Power Wide Area Network (LPWAN) [Centenaro et al. 2016]. Uma LPWAN tem uma taxa de transferência baixa, mas pode cobrir áreas de até 50 quilômetros. Entre as tecnologias e protocolos LPWAN destaca-se o padrão aberto LoRaWAN que opera sobre a tecnologia de radiofrequência LoRa. 
Diversas aplicações IoT contam com o uso de um sistema de posicionamento global, Global Positioning System (GPS), para rastrear um veículo em movimento dentro de uma cidade e armazenar o histórico do seu percurso, por exemplo. O GPS oferece localização precisa em tempo real, porém consome muita energia, uma vez que o processamento da informação ocorre no dispositivo [Manzoni et al. 2019]. Além disso a falha de um GPS resulta na inoperabilidade da aplicação.

Existem diversas abordagens de localização que dependem apenas do sinal recebido da aplicação. Entre essas abordagens estão as medidas de distância como por tempo de chegada (Time of Arrival, ToA), tempo de voo (Time of Flight, ToF), indicador de potência do sinal recebido (Received Signal Strength Indication, RSSI), e diferença de tempo de chegada (Time Difference of Arrival, TDoA), ou até por medida de ângulo como ângulo de chegada (Angle of Arrival, AoA) [Khelifi et al. 2019]. Essas abordagens utilizam diferentes técnicas para estimar a posição de um objeto dependendo do número de dispositivos envolvidos, sendo as mais comuns triangulação, trilateração e multilateração. Para isso, utilizam duas bases receptoras, três e mais de três, respectivamente, para calcular a posição de um objeto emissor [Fargas and Petersen 2017].

Técnicas de tolerância a falhas em IoT visam garantir o funcionamento correto do sistema mesmo na ocorrência de falhas e são geralmente baseadas em redundância, exigindo componentes adicionais ou algoritmos especiais [Pastório et al. 2020]. O objetivo deste trabalho é propor e avaliar uma solução de tolerância a falhas para um sistema de geolocalização baseado em GPS que faz uso de uma rede LoRaWAN. A solução faz uso de técnicas baseadas em medidas de distância RSSI e TDoA para estimar a nova posição do objeto em caso de falha do GPS. A solução foi avaliada em um ambiente com três bases receptoras instaladas em um bairro da cidade de Toledo/PR. Os algoritmos são implementados e comparados com a ferramenta comercial LoRa Cloud, que permite apenas mil requisições gratuitas diariamente. Os resultados demonstram erros inferiores a $151 \mathrm{e}$ 126 metros no pior caso e 19 e 11 metros no melhor caso considerando a implementação realizada e a ferramenta LoRa Cloud, respectivamente.

Esta proposta surgiu de uma parceria envolvendo o município e a universidade que visa desenvolver uma aplicação para rastrear caminhões de coleta seletiva de lixo no conceito de cidades inteligentes. Os caminhões utilizam de GPS para enviar as suas coordenadas geográficas para a rede através da rede LoRaWAN já instalada na universidade [Rossato et al. 2020]. Ocorre em alguns momentos a perda de conexão do GPS com o satélite, gerando a falha total do sistema, sendo impossível identificar a posição do caminhão até recuperar conexão GPS. Em uma revisão sistemática da literatura realizada em [Pastório et al. 2020] sobre tolerância a falhas em IoT e em outras buscas na literatura especializada não se encontrou trabalho que aborde a falha do GPS em redes LPWAN.

A organização do trabalho segue da seguinte forma. A Seção 2 apresenta a rede LoRa/LoRaWAN, os conceitos de geolocalização e alguns trabalhos relacionados. A Seção 3 descreve a solução proposta e metodologia. Os resultados obtidos se encontram na Seção 4. Por fim, a conclusão e os trabalhos futuros estão na Seção 5.

\section{LoRa/LoRaWAN e Geolocalização}

Esta seção apresenta a definição de LoRa e LoRaWAN. Os conceitos e técnicas de geolocalização são apresentados na sequência. Por fim são expostos alguns trabalhos 
que tem a geolocalização como tema principal.

\subsection{LoRa e LoRaWAN}

LoRa é uma tecnologia de radiofrequência (RF) proprietária da empresa Semtech. Permite enviar dados por longas distâncias, com baixo consumo de energia e baixas taxas de transmissão. LoRa utiliza a técnica de modulação Chiper Spread Spectrum (CSS) e opera em bandas de radiofrequência não licenciadas. No Brasil o padrão utilizado é o mesmo da Austrália, na faixa de $915 \mathrm{MHz}$, compreendendo a faixa de $902 \mathrm{MHz}$ a 907,5 MHz e $915 \mathrm{MHz}$ a $928 \mathrm{MHz}$.

Para configuração de dispositivos finais que compõem uma rede LoRa, uma das principais variáveis a ser definida é o fator de espalhamento, Spreading Factor (SF), [de Oliveira et al. 2018]. O SF é um valor discreto, de 7 a 12, e influência na duração da modulação interferindo nos valores de bit rate (quantidade de bits por segundo) e air time (tempo em que o pacote está no ar).

LoRaWAN é um protocolo de comunicação de rede que tem LoRa como camada física [LORA ALLIANCE 2015]. A Figura 1 mostra a topologia típica de uma rede LoRaWAN. Onde os dispositivos finais, são dispositivos que através de sensores e atuadores monitoram e atuam no ambiente. O gateway, responsável por conectar os dispositivos LoRa com a Internet repassando a informação obtida para o servidor. A aplicação utiliza de serviços fornecidos pelo servidor para exibir informações e, ou, fornecer funcionalidades.

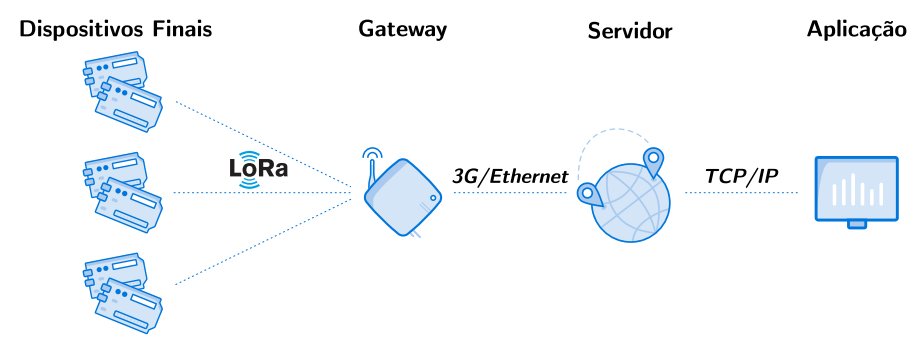

Figura 1. Topologia de uma rede LoRaWAN.

\subsection{Geolocalização}

Sistemas de RF realizam a troca de mensagens por ondas eletromagnéticas que viajam pelo ar com mudanças em amplitude e frequência. Conhecidas as características da onda é possível identificar o local da fonte de transmissão. As técnicas baseadas em medidas de distância RSSI e TDoA são descritas nesta seção. Estas técnicas foram escolhidas pois suas características estão presentes na rede LoRa. As deduções matemáticas são feitas com a multilateração como referência, dessa forma podendo ser generalizada para uma estratégia de trilateração.

\subsection{Indicador de Potência do Sinal Recebido - RSSI}

O indicador de potência do sinal recebido, RSSI, fornece informação sobre a qualidade do sinal que está sendo recebido. Conhecendo este valor e o modelo de propagação do sinal é possível estimar distância entre o transmissor e o receptor [Gu et al. 2018]. A Figura 2 demonstra as circunferências obtidas através do cálculo de distância por RSSI de três gateways. Percebe-se que no ponto de intersecção entre elas tem-se o emissor. 


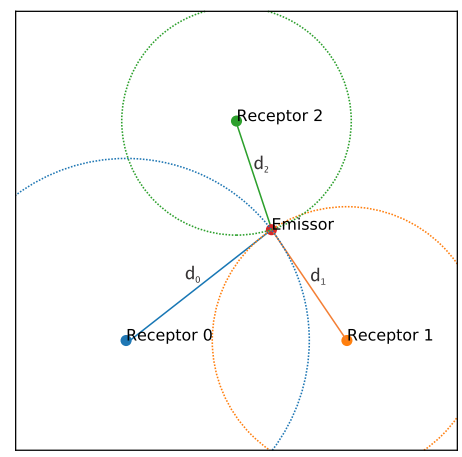

Figura 2. Circunferências de distância calculadas com RSSI.

A distância entre o transmissor e o receptor pode ser calculada através da Equação 1, que é derivada de um modelo empírico [Manzoni et al. 2019]. Onde $d$ é a distância entre receptor e emissor, $R S S I$ é o indicador de potência do sinal, e $n$ é um valor experimental, normalmente entre 2 e 6 , que representa o fator de perda de sinal.

$$
d=10^{-R S S I / 10 n}
$$

Utilizando Pitágoras a distância entre o emissor e os receptores pode ser representada pela Equação 2. Onde $d_{i}$ é a distância entre o emissor e o receptor $i,(x, y)$ é a posição do emissor, e $\left(x_{i}, y_{i}\right)$ é a posição do receptor $i$.

$$
d_{i}=\sqrt{\left(x-x_{i}\right)^{2}+\left(y-y_{i}\right)^{2}}=10^{-R S S I / 10 n}
$$

Rearranjando os membros obtém-se a Equação 3, formando um sistema de equações. A solução deste sistema de equações resulta em uma posição $(x, y)$ correspondente ao emissor.

$$
\sqrt{\left(x-x_{i}\right)^{2}+\left(y-y_{i}\right)^{2}}-d_{i}=0
$$

\subsection{Diferença do Tempo de Chegada - TDoA}

Diferença do tempo de chegada, TDoA, é uma técnica de estimativa de posição baseada em hipérboles. Para estimar a localização de um emissor, faz-se necessário pelo menos três bases de recebimento [Bissett 2018], ou seja, gateways, como visto na Figura 3. A Figura 3a mostra as distância de cada receptor para o emissor. Já a Figura 3b apresenta as hipérboles formadas pelo Receptor 0 , que recebeu o sinal primeiro.

Dessa forma, para estimar a posição do emissor $(x, y)$, tendo $n$ bases receptoras de posição $\left(X_{i}, Y_{i}\right), i=0, . ., n-1$. A Equação 4 representa a diferença de distância entre bases de recebimento $d_{i}$ e $d_{j}$, com $j \neq i$, onde $C$ é a velocidade da luz, $t_{i}$ e $t_{j}$ tempos de chegada nas respectivas bases de recebimento.

$$
\Delta d_{i, j}=C\left(t_{i}-t_{j}\right)=d_{i}-d_{j}
$$

Utilizando Pitágoras a distância entre o transmissor e os receptores pode ser representada pela Equação 5. 


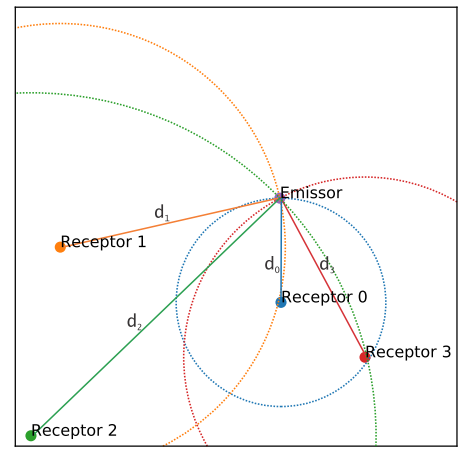

(a)

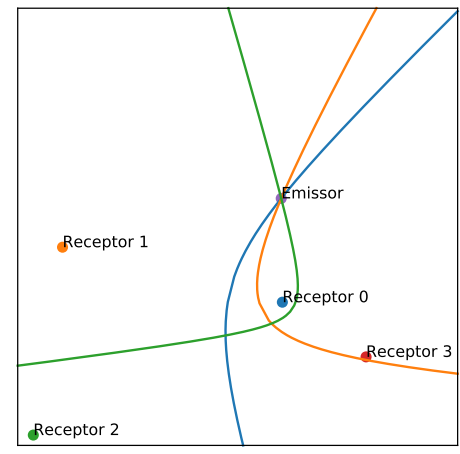

(b)

Figura 3. Multilatereção por TDoA. (a) Distâncias dos receptores para o emissor. (b) Hipérboles formadas pelo Receptor 0.

$$
d_{i}=\sqrt{\left(x-X_{i}\right)^{2}+\left(y-Y_{i}\right)^{2}}
$$

Fixando como âncora a base que recebeu o sinal primeiro e combinando as equações 4 e 5 , têm-se um conjunto de equações de segundo grau, que podem ser vistas na Equação 6. Onde $\left(x_{a}, y_{a}\right)$ é a posição da base que recebeu o sinal primeiro, $\left(x_{j}, y_{j}\right), j \neq i$ a posição das outras bases, $t_{a}$ o tempo de recebimento na âncora e $t_{j}, j \neq i$ o tempo de recebimento das outras bases.

$$
\sqrt{\left(x-x_{a}\right)^{2}+\left(y-y_{a}\right)^{2}}-\sqrt{\left(x-x_{i}\right)^{2}+\left(y-y_{i}\right)^{2}}+C\left(t_{i}-t_{a}\right)=0
$$

\subsection{Trabalhos Relacionados}

O trabalho de [Podevijn et al. 2018b] utiliza uma rede LoRa pública para realizar o rastreamento de dispositivos. Através de cálculos TDoA foi possível estimar a posição do dispositivo em diferentes cenários durante uma caminhada ou dirigindo. Os resultados apresentaram uma precisão média de 200 metros. Este trabalho evoluiu para o trabalho em [Podevijn et al. 2018a] onde o algoritmo de geolocalização passar a considerar a infraestrutura das ruas e a velocidade do dispositivo. Com isso, a precisão aumentou para uma média de 75 metros. Já Manzoni et. al, [Manzoni et al. 2019] realiza a geolocalização de veículos em um ambiente interno usando uma rede LoRa. Com uma arquitetura formada por 3 gateways a localização dos veículos é estimada pela potência de sinal recebido, RSSI. Foram realizados experimentos em um estacionamento obtendo precisão de até 20 metros.

A localização de um dispositivo estático é realizada utilizando TDoA em uma rede LoRa em [Fargas and Petersen 2017]. Por o dispositivo não apresentar movimento o trabalho pôde realizar várias medidas para estimar o seu local. Dessa forma, foi possível retirar os pontos discrepantes que poderiam causar um eventual erro. $\mathrm{O}$ trabalho conseguiu estimar a posição do dispositivo com uma média de 100 metros de precisão.

Percebe-se uma precisão variável nos trabalhos relacionados a geolocalização de acordo com o cenário e contexto propostos. Por exemplo, em um ambiente interno a precisão é maior se comparada a um ambiente externo, mas talvez a precisão relativa 
seja parecida. Observa-se também o uso de diferentes técnicas para alcançar uma maior precisão. No entanto, os trabalhos não explorar técnicas de geolocalização perante a falha permanente ou temporária do módulo GPS.

\section{Solução Tolerante a Falhas para Geolocalização}

Esta seção apresenta a metodologia utilizada durante o desenvolvimento do trabalho e descreve a solução proposta.

\subsection{Dispositivos}

O dispositivo responsável por realizar o rastreamento dos caminhões da coleta seletiva de lixo é o TTGO T-BEAM, que já teve o seu desempenho avaliado na rede LoRaWAN da UTFPR [Almeida et al. 2020]. O dispositivo é construído em torno do microprocessador ESP32, e conta com as funcionalidades dos módulos integrados, GPS e LoRa. A programação do T-BEAM pode ser feita em C, C++ e Python. O protocolo LoRa disponível para o dispositivo é fornecido por firmware e distribuído através da biblioteca LMIC.

Foram utilizados três gateways compostos por um Raspberry Pi 3 B+, um módulo HAT e concentrador comercial LoRaWAN da empresa Radioenge. Os módulos possuem GPS integrado, o que possibilita a sincronia de tempo entre os dispositivos com uma precisão de microssegundos. Os gateways se conectam à rede através da The Things Network (TTN). TTN é uma rede colaborativa que conecta gateways LoRaWAN [THE THINGS NETWORK 2020]. É uma plataforma gratuita e aberta que conta com várias funcionalidades e integrações a serem utilizadas por aplicações desenvolvidas.

\subsection{Geolocalização}

Os algoritmos de geolocalização são implementados em Python. Além disso, as distâncias são estimadas através da plataforma LoRa Cloud que realizam os cálculos utilizando RSSI e TDoA. LoRa Cloud é uma plataforma da Semtech que oferece o serviço de geolocalização através de uma API. O serviço grátis da plataforma permite mil requisições diárias. Os resultados das duas soluções de geolocalização serão comparados de forma a avaliar a eficiência da implementação proposta.

\subsection{Solução Tolerante a Falhas}

A solução consiste em envios periódicos de localização dos caminhões para a rede através da rede LoRa. Em casos em que o GPS não recebe sinal do satélite um valor nulo é enviado, deixando explícito que o cálculo de geolocalização é necessário. A posição do caminhão é estimada utilizando algoritmos de geolocalização e então é enviada para visualização no mapa. O fluxograma da proposta pode ser visualizado na Figura 4. Primeiramente, identificando se o pacote contém a coordenada proveniente do GPS. Então a próxima etapa verifica se 3 ou mais gateways receberam o mesmo pacote. Se sim, é necessário a conversão das localizações angulares dos gateways, latitude e longitude, para o sistema cartesiano. Isto é feito utilizando o caso especial plate carrée da projeção equirretangular [Kumler 1994], cuja equação pode ser vista na Equação 7, onde $R$ é o raio do planeta Terra, lng e lat longitude e latitude a serem convertidas, $x$ e $y$ resultado da conversão, e $l n g_{0}$ e $l a t_{0}$ a latitude e longitude do ponto central do mapa. A visualização dos dados ocorre pelo framework Leaflet com OpenStreetMap. 


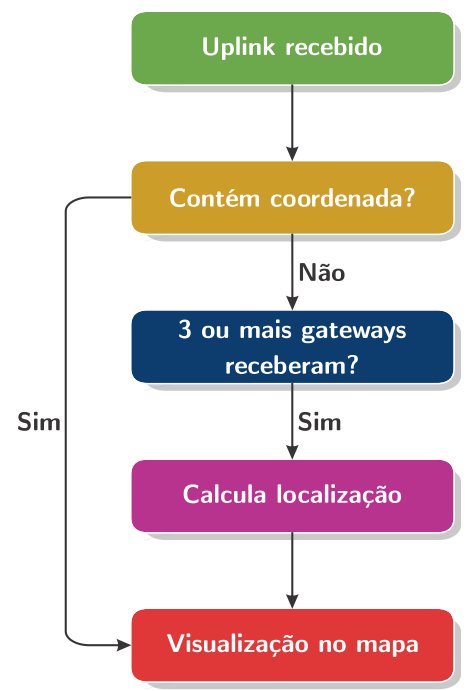

Figura 4. Fluxo de execução da proposta.

$$
\begin{aligned}
& x=R\left(\operatorname{lng}-\operatorname{lng}_{0}\right) \\
& y=R\left(l a t-l a t_{0}\right)
\end{aligned}
$$

O algoritmo que recebe as três coordenadas pode realizar o calculo de geolocalização com as equações de TDoA (de acordo com Equação 6, Seção 2.4) e RSSI (de acordo com Equação 3, Seção 2.3). O algoritmo faz uso da biblioteca SciPy para realizar a aproximação numérica dessas equações por mínimos quadrados e uma implementação de série de Taylor pelo método de Foy [FOY 1976]. Esta implementação faz parte do trabalho de [Crowell 2018], o qual foi utilizado e alterado durante este trabalho. A esta implementação foram adicionados os métodos de mínimos quadrados e RSSI ao algoritmo.

As coordenadas cartesianas estimadas podem ser revertidas para latitude e longitude e exibidas no mapa. Por fim, para os pacotes que possuem as coordenadas GPS no seu payload é possível calcular o erro entre GPS e calculado utilizando a fórmula inversa de Haversine, Equação 8 de [Nichat 2013], onde $R$ é o raio do planeta Terra, lat e lng são as respectivas latitudes e longitudes dos pontos a serem calculadas as distâncias em radianos, e $d$ a distância resultante.

$$
d=2 R \cdot \operatorname{arcsen} \sqrt{\operatorname{sen}^{2}\left(\frac{\operatorname{lat}_{2}-l a t_{1}}{2}\right)+\cos \left(l a t_{1}\right) \cos \left(l a t_{2}\right) \operatorname{sen}^{2}\left(\frac{\operatorname{lng}_{2}-l n g_{1}}{2}\right)}
$$

\section{Análise e Discussão dos Resultados}

Esta seção apresenta o ambiente de teste com a metodologia indicada anteriormente. Os resultados incluem uma comparação com o algoritmo de geolocalização implementado em Python com a ferramenta comercial LoRa Cloud. A seguir são descritos o ambiente de teste, os resultados com diferentes fatores de espalhamento e as considerações sobre os resultados. 


\subsection{Ambiente de Testes}

Os gateways foram posicionados em áreas externas de duas residências localizadas em um bairro da cidade de Toledo, de maneira a tentar cobrir a maior área entre as residências. As posições das antenas de cada gateway podem ser vista na Figura 5. Percebe-se pelas imagens que as antenas não puderam ser posicionadas perfeitamente, altura baixa e obstáculos ao redor. Na Figura 6 estão presentes as localizações dos gateways em azul e o caminho a ser percorrido durante os testes em vermelho. Nesta imagem pode se perceber que os gateways 1 e 2 estão na mesma residência. Os gateways contam ainda com um GPS cada, item essencial para sincronizar o tempo entre os equipamentos.

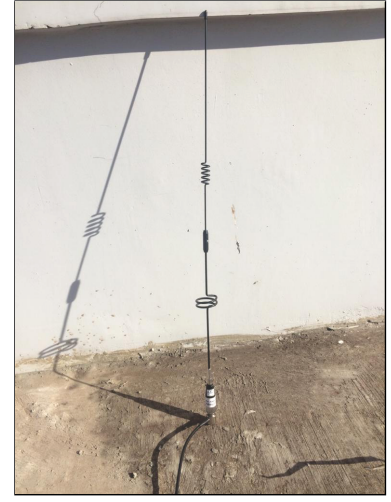

(a) gateway 1

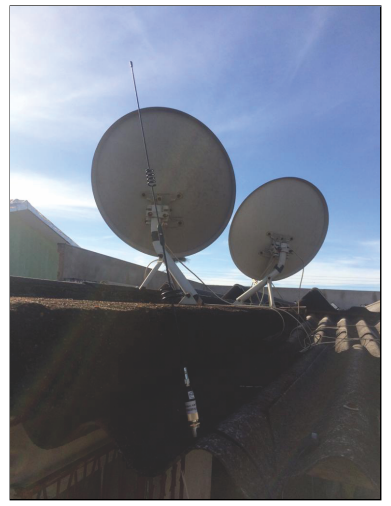

(b) gateway 2.

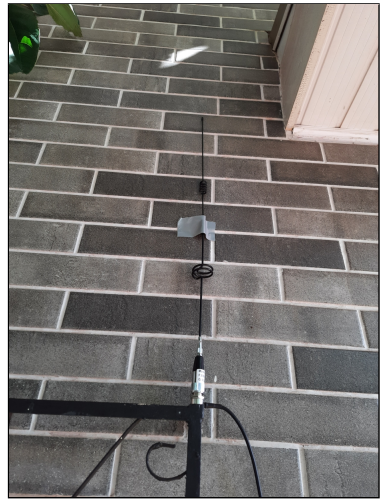

(c) gateway 3 .

Figura 5. Posições das antenas dos gateways durante os testes.

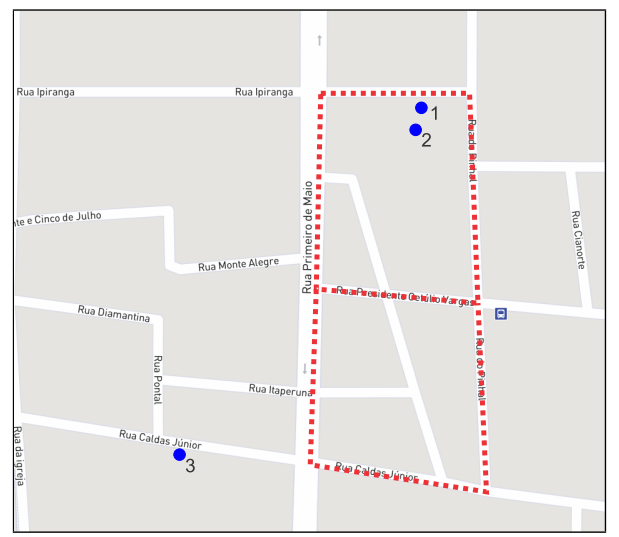

Figura 6. Percurso (vermelho) e localização dos gateways (azul).

O dispositivo T-Beam com o firmware de rastreamento, escrito em $\mathrm{C}++$ com LMIC, foi levado a esta área para serem realizados os testes. O T-Beam foi configurado para enviar pacotes a cada 30 segundos. A avaliação aconteceu com 2 SFs diferentes, SF7 e SF10, em momentos distintos. Foram escolhidas duas configurações de SF diferentes de forma a identificar o seu impacto nos resultados. O dispositivo foi levado em caminhadas realizando o percurso da Figura 6 de forma a simular o funcionamento do caminhão, obtendo os dados necessários para estimar a sua posição. 


\subsection{Resultados com SF7}

Esta seção apresenta os resultados obtidos com o parâmetro de transmissão em SF7. SF7 é uma configuração indicada para pequenas distâncias possuindo a maior taxa de transmissão de dados dentre as configurações possíveis. Foram 27 pacotes recebidos, destes somente 9 foram recebidos pelos 3 gateways. A partir destes 9 pacotes foi possível estimar a posição do dispositivo, mas o erro só pôde ser calculado em 7, pois continham a posição do dispositivo GPS no payload.

Nas Figuras 7 e 8 é possível ver os pontos calculados por TDoA e RSSI, respectivamente, junto com os pontos do GPS do dispositivo que foram recebidos pelos 3 gateways. As figuras apresentam os dois métodos de aproximação utilizados, mínimos quadrados em (a) e série de Taylor em (b). Em vermelho as posições calculadas por TDoA, em roxo por RSSI e em verde as posições recebidas do GPS.

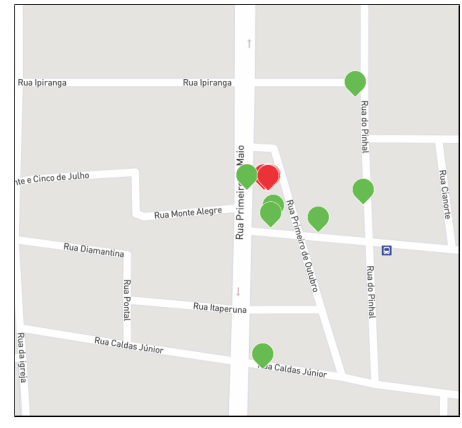

(a) Mínimos quadrados.

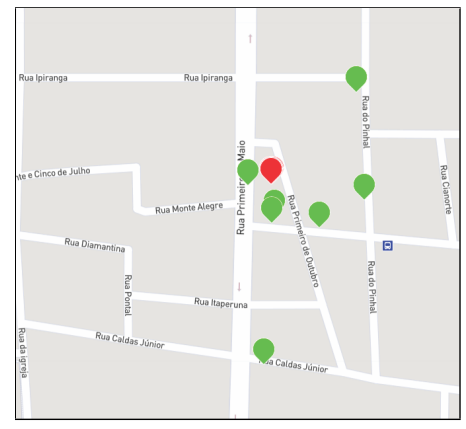

(b) Série de Taylor.

Figura 7. Posições por TDoA (vermelho) e GPS (verde) em SF7.

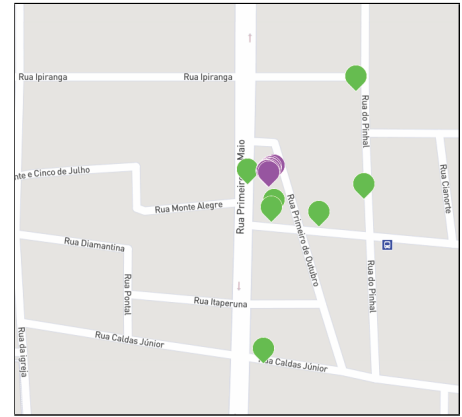

(a) Mínimos quadrados.

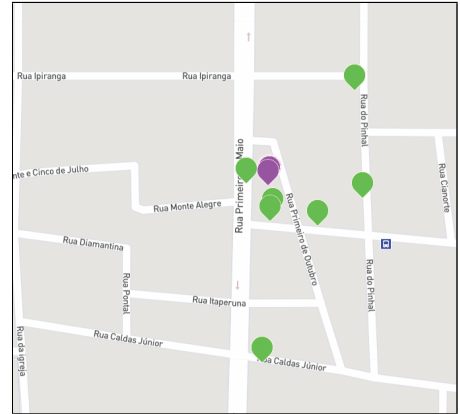

(b) Série de Taylor.

Figura 8. Posições por RSSI (roxo) e GPS (verde) em SF7.

As localizações obtidas da LoRa Cloud podem ser visualizadas na Figura 9 na cor laranja. LoRa Cloud utiliza seus próprios métodos de decisão de algoritmos. É possível especificar qual método a ser utilizado, TDoA, RSSI ou uma combinação dos dois. Mas a plataforma escolhe o método que considera ter maior precisão independente do especificado.

Buscando os resultados numéricos das posições calculadas foi realizada a média do erro encontrado em cada amostra, conforme Equação 8 Seção 3. A Tabela 1 apresenta os resultados obtidos através de TDoA e RSSI com a transmissão de dados em 


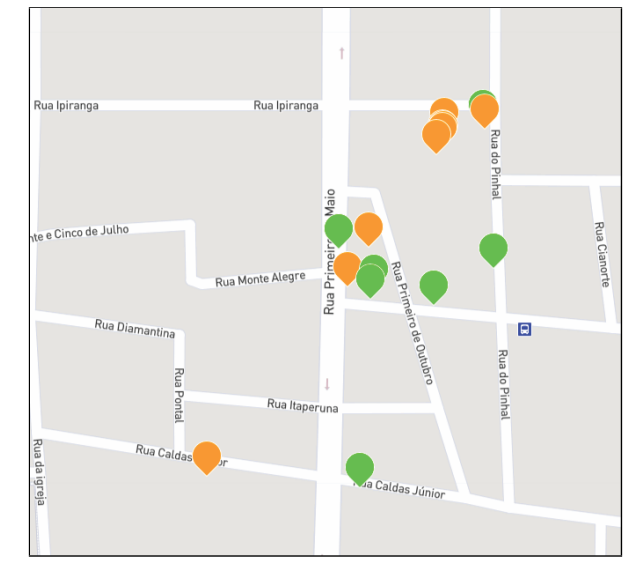

Figura 9. Posições por LoRa Cloud (laranja) e GPS (verde) em SF7.

SF7. A tabela compara a precisão média dos resultados, junto da melhor e pior precisão em cada modelo de aproximação numérica, mínimos quadrados e série de Taylor. Percebendo-se que em TDoA a precisão foi maior utilizando mínimos quadrados, já em RSSI a aproximação por série de Taylor apresentou uma média e pior caso melhores.

Tabela 1. Precisão do algoritmo de TDoA e RSSI em SF7 em metros.

\begin{tabular}{|r|r|r|r|r|}
\hline & \multicolumn{2}{|c|}{ TDoA } & \multicolumn{2}{c|}{ RSSI } \\
\hline & Mínimos quadrados & Série de Taylor & Mínimos quadrados & Série de Taylor \\
\hline Média & 65,47 & 65,83 & 66,66 & 65,40 \\
\hline Melhor & 18,22 & 19,89 & 18,38 & 19,89 \\
\hline Pior & 147,08 & 148,89 & 150,41 & 145,40 \\
\hline
\end{tabular}

A Tabela 2 compara os resultados obtidos em TDoA e RSSI com os recebidos da plataforma LoRa Cloud. Apresentam-se os melhores resultados obtidos comparando-os com os resultados calculados pela ferramenta LoRa Cloud. Observa-se que RSSI teve a melhor média, mas o melhor caso ocorre em TDoA com uma precisão de 18,22 metros.

Tabela 2. Comparação dos resultados em SF7 em metros.

\begin{tabular}{|r|r|r|r|}
\hline & TDoA & RSSI & LoRa Cloud \\
\hline Média & 65,47 & 65,40 & 79,60 \\
\hline Melhor & 18,22 & 18,38 & 28,55 \\
\hline Pior & 148,89 & 150,41 & 123,83 \\
\hline
\end{tabular}

Os resultados apresentaram uma boa precisão no melhor caso, chegando a aproximadamente 19 metros em RSSI e TDoA. No pior caso os resultados apresentam precisam aproximada de 149 e 151 metros para TDoA e RSSI, respectivamente. Ao comparar visualmente os mapas, percebe-se que os pontos calculados pelo algoritmo de aproximação estão sobrepostos. Ao contrário, os pontos calculados pela LoRa Cloud estão mais dispersos. Tal fato indica um possível enviesamento dos cálculos devido a posição dos gateways.

\subsection{Resultados com SF10}

SF10 é uma configuração de transmissão intermediária, indicada para médias distâncias com uma taxa de transmissão menor do que em SF7. Tendo em consideração esta característica, foram 78 pacotes recebidos destes, 20 foram recebidos pelos 3 gateways. 
As localizações obtidas dos cálculos de geolocalização podem ser vistas nas Figuras 10, TDoA em vermelho, e 11, RSSI em roxo, junto com as posições recebidas pelo GPS, em verde. Da mesma forma apresentada nos resultados de SF7, em (a) aproximação por mínimos quadrados e em (b) por série de Taylor. Já na Figura 12 podem ser visualizados os pontos recebidos da LoRa Cloud, em alaranjado, e do GPS, em verde.

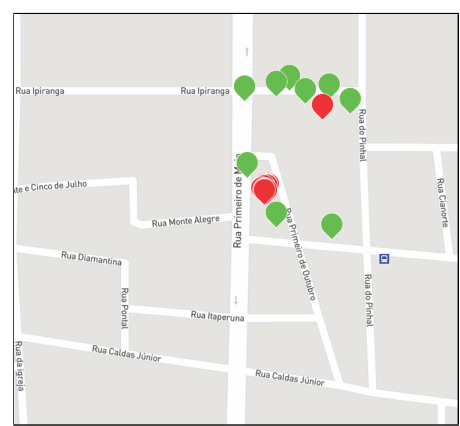

(a) Mínimos quadrados.

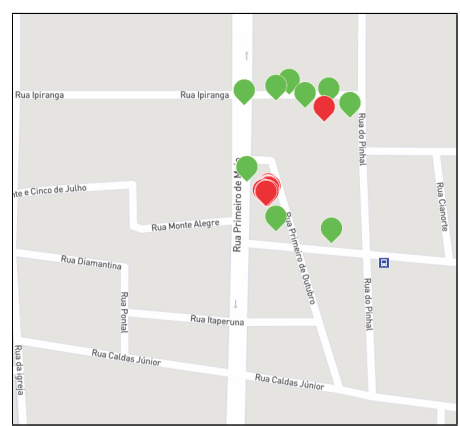

(b) Série de Taylor.

Figura 10. Posições por TDoA (vermelho) e GPS (verde) em SF10.

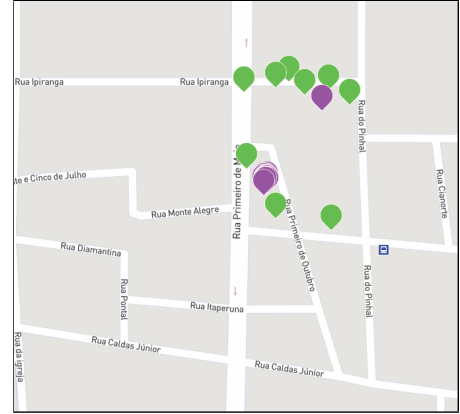

(a) Mínimos quadrados.

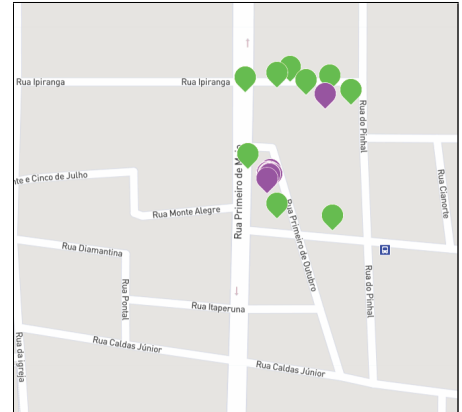

(b) Série de Taylor.

Figura 11. Posições por RSSI (roxo) e GPS (verde) em SF10.

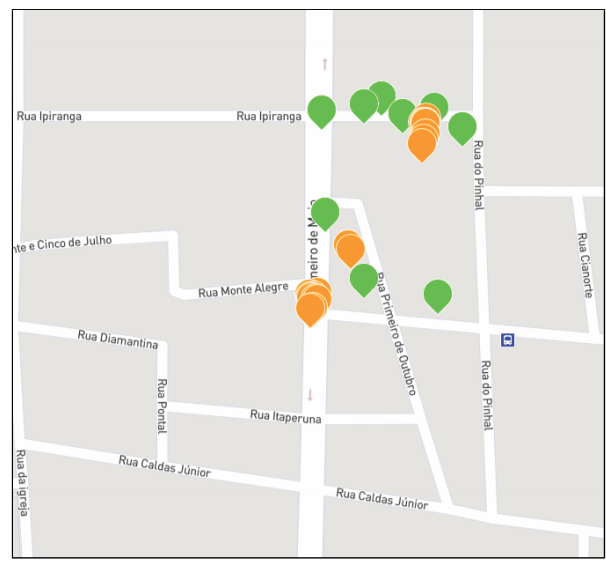

Figura 12. Posições por LoRa Cloud (laranja) e GPS (verde) em SF10.

A média dos erros, melhor e pior caso, são apresentados na Tabela 3 para TDoA e RSSI. Nesta configuração, a série de Taylor superou o método de mínimos quadrados 
Tabela 3. Precisão do algoritmo de TDoA e RSSI em SF10 em metros.

\begin{tabular}{|r|r|r|r|r|}
\hline & \multicolumn{2}{|c|}{ TDoA } & \multicolumn{2}{c|}{ RSSI } \\
\hline & Mínimos quadrados & Série de Taylor & Mínimos quadrados & Série de Taylor \\
\hline Média & 73,22 & 71,71 & 72,79 & 70,50 \\
\hline Melhor & 23,64 & 24,85 & 21,61 & 22,23 \\
\hline Pior & 98,26 & 95,90 & 99,77 & 96,07 \\
\hline
\end{tabular}

para caso médio e o pior caso tanto para TDoA quanto para RSSI. Já para o melhor caso, a diferença foi pequena em relação a aproximação por mínimos quadrados.

Os resultados obtidos com os algoritmos de TDoA e RSSI são comparados com os recebidos da LoRa Cloud na Tabela 4. Observa-se que LoRa Cloud obteve os melhores resultados na média e melhor caso, chegando a uma precisão em cerca de 11 metros. Porém, no pior caso o resultado foi inferior aos outros métodos.

Tabela 4. Comparação dos resultados em SF10 em metros.

\begin{tabular}{|r|r|r|r|}
\hline & TDoA & RSSI & LoRa Cloud \\
\hline Média & 71,71 & 70,50 & 66,14 \\
\hline Melhor & 23,64 & 21,61 & 10,94 \\
\hline Pior & 98,26 & 99,77 & 125,81 \\
\hline
\end{tabular}

\subsection{Considerações Sobre os Resultados}

Os resultados dos algoritmos de geolocalização com TDoA e RSSI foram muito semelhantes, tanto em SF7 quanto em SF10. Mesmo ao comparar os resultados de TDoA com RSSI a diferença é pequena, nas médias aproximadamente 1 metro. Um dos fatores que influenciaram nos resultados de geolocalização com TDoA foi a precisão dos relógios dos gateways. Como a velocidade de propagação do sinal RF LoRa se compara a velocidade da luz (299.792.458 metros por segundo) e com a precisão de tempo dos gateways em 1 microssegundo, existe um erro embutido nos cálculos de no mínimo 300 metros, pois a cada nanosegundo 1 metro de erro é adicionado. Este foi um parâmetro descoberto durante a experimentação, já que a documentação dos dispositivos não indicava tal característica.

Mesmo com a baixa precisão de tempo dos gateways não foram identificados erros maiores que 151 metros. Acontece que como 2 dos 3 gateways se encontravam relativamente próximos, muitas vezes o tempo de chegada do pacote era o mesmo, fazendo com que o sistema de equações tivesse praticamente, uma equação a menos. Em outros casos, o tempo de chegada era muito parecido em todos os gateways. Dessa forma, as equações aproximavam-se muito a equação de uma circunferência, explicando o motivo dos cálculos resultarem sempre em um ponto central do mapa.

No caso dos cálculos por RSSI, identificou-se pouca influência do fator $n$ presente no modelo apresentado. Tal fator foi alterado durante os experimentos, no entanto chegouse aos mesmos resultados. Porém, no caso do RSSI, era esperado um comportamento parecido com o obtido devido ao posicionamento dos gateways.

Em se tratando da ferramenta LoRa Cloud, apesar de ter sido especificado nas requisições o calculo de geolocalização por TDoA, a maior parte dos resultados retornaram como RSSI. Com isso alguns pontos apresentaram uma boa precisão, chegando em 
até 11 metros de precisão durante a transmissão em SF10. Avaliando os demais resultados, em SF7 LoRa Cloud apresentou um erro médio maior, se comparado com os demais algoritmos, mas um intervalo de erro menor, entre o melhor e o pior. Comportamento contrário ao que ocorreu em SF10, em que a precisão média da LoRa Cloud é a melhor.

\section{Conclusão}

Este trabalho apresentou uma proposta para mitigar a falha do módulo GPS em dispositivos IoT que operam em uma rede de LoRaWAN. As técnicas de geolocalização TDoA e RSSI foram aplicadas de forma a se estimar a posição de um objeto em movimento perante a falha do GPS. Através de envios periódicos de mensagens o dispositivo pode ser rastreado por GPS e, no caso de falha do GPS, a sua posição pode ser estimada através dos algoritmos de aproximação. Os algoritmos implementados em Python foram comparados à ferramenta comercial LoRa Cloud. A apresentação dos dados foi implementada em uma aplicação Web, de forma a visualizar os dados. Um cenário de testes com 3 gateways foi construído em um bairro da cidade de Toledo.

A abordagem proposta aproveita o contexto da aplicação para não impor carga computacional adicional ao dispositivo IoT. Os cálculos para se estimar a posição do objetos são executados na nuvem. Avaliando os resultados obtidos foi identificado que utilizar técnicas de geolocalização pode ser uma alternativa para o rastreamento de um objeto com precisão de até 151 metros. Dessa forma, aplicações em que a posição do objeto não precisa ser extremamente exata, podem utilizar desta proposta para adquirir tolerância a falhas em seu funcionamento.

Como trabalhos futuros, é possível aprimorar a implementação como no trabalho em [Podevijn et al. 2018a]. O trabalho realiza o ajuste das localizações estimadas de acordo com o objeto que está sendo rastreado. Por exemplo, se está sendo rastreado um veículo espera-se que as posições estimadas, e recebidas, estejam localizadas em alguma rua ou rodovia. Uma solução parecida com a proposta neste trabalho pode ser implementada utilizando a fórmula inversa de Haversine. As equações de TDoA e RSSI são basicamente cálculos de distâncias, substituir o termo em raiz quadrada pela fórmula inversa de Haversine permite a aproximação numérica diretamente pela latitude e longitude, sem necessidade de conversão para coordenadas cartesianas. Isto pode vir a aumentar a precisão do algoritmo, pois evita a propagação do erro entre conversões. Outra alternativa é o uso de aprendizado de máquina para realizar os cálculos de geolocalização. A rede neural pode ser treinada utilizando os dados de ToA e RSSI e até mesmo as posições recebidas e estimadas anteriormente para calcular a posição do emissor. Além disso, ampliar o ambiente de testes envolvendo mais bairros ou até mesmo toda a cidade a fim de avaliar com mais profundidade a eficiência da proposta.

\section{Referências}

Almeida, T. V. O., Silva, M., Souza, J., Souza, A. R., , Spanhol, F. A., and Camargo, E. T. (2020). Avaliação de dispositivos de rastreamento em uma rede lorawan no contexto de cidades inteligentes. In Anais do IV Workshop de Computação Urbana, pages 1-14, Porto Alegre, RS, Brasil. SBC.

Bissett, D. (2018). Analysing tdoa localisation in lora networks. Master's thesis, Delft University of Technology. 
Centenaro, M., Vangelista, L., Zanella, A., and Zorzi, M. (2016). Long-range communications in unlicensed bands: the rising stars in the iot and smart city scenarios. IEEE Wirel. Comm., 23(5):60-67.

Crowell, C. (2018). Lorawan geolocalization using tdoa. Master's thesis, University of New Hampshire.

de Oliveira, L., da Conceição, A., and Neto, L. S. (2018). Revisão sistemática da literatura sobre aplicações das tecnologias lora e lorawan. In Anais Estendidos do VIII Simpósio Brasileiro de Engenharia de Sistemas Computacionais, Porto Alegre, RS, Brasil. SBC.

Fargas, B. C. and Petersen, M. N. (2017). Gps-free geolocation using lora in low-power wans. In 2017 Global Internet of Things Summit (GIoTS), pages 1-6.

FOY, W. H. (1976). Position-location solutions by taylor-series estimation. IEEE Transactions on Aerospace and Electronic Systems, AES-12(2):187-194.

Gu, C., Jiang, L., and Tan, R. (2018). Lora-based localization: Opportunities and challenges. CoRR, abs/1812.11481.

Khelifi, F., Bradai, A., Benslimane, A., Rawat, P., and Atri, M. (2019). A survey of localization systems in internet of things. Mobile Networks and Applications, 24(3):761785 .

Kumler, M. (1994). Flattening the earth: Two thousand years of map projections. Cartographic Perspectives, (18):32-33.

LORA ALLIANCE (2015). Lorawan: What is it? a technical overview of lora and lorawan. acesso em 22/09/2020.

Manzoni, P., Calafate, C. T., Cano, J.-C., and Hernández-Orallo, E. (2019). Indoor vehicles geolocalization using lorawan. Future Internet, 11(6):124.

Nichat, M. (2013). Landmark based shortest path detection by using a* algorithm and haversine formula.

Pastório, A. F., Camargo, E. T., and Rodrigues, L. A. (2020). Uma revisão sistemática da literatura sobre tolerância a falhas em internet das coisas. In Anais Estendidos do X Simpósio Brasileiro de Engenharia de Sistemas Computacionais, pages 57-64, Porto Alegre, RS, Brasil. SBC.

Podevijn, N., Plets, D., Trogh, J., Martens, L., Suanet, P., Hendrikse, K., and Joseph, W. (2018a). Tdoa-based outdoor positioning with tracking algorithm in a public lora network. Wireless Communications and Mobile Computing, 2018:1864209.

Podevijn, N., Trogh, J., Karaagac, A., Haxhibeqiri, J., Hoebeke, J., Martens, L., Suanet, P., Hendrikse, K., Plets, D., and Joseph, W. (2018b). Tdoa-based outdoor positioning in a public lora network. In 12th European Conference on Antennas and Propagation (EuCAP 2018), pages 1-4.

Rossato, J., Spanhol, F. A., and Camargo, E. T. (2020). Implantação e avaliação de uma rede sem-fio de longo alcance e baixa potência para cidades inteligentes. In Anais do IV CoUrb / IV Workshop de Computação Urbana, pages 1-14, Porto Alegre, RS, Brasil. SBC.

THE THINGS NETWORK (2020). The things network (ttn). acesso em 22/09/2020. 